\title{
New Subclasses of Analytic Functions with Respect to Symmetric and Conjugate Points
}

\author{
Huo Tang ${ }^{1,2}$ and Guan-Tie Deng ${ }^{1}$ \\ ${ }^{1}$ School of Mathematical Sciences, Beijing Normal University, Beijing 100875, China \\ ${ }^{2}$ School of Mathematics and Statistics, Chifeng University, Chifeng, Inner Mongolia 024000, China \\ Correspondence should be addressed to Huo Tang; thth2009@tom.com
}

Received 22 September 2012; Accepted 11 November 2012

Academic Editor: Jacek Dziok

Copyright (c) 2013 H. Tang and G.-T. Deng. This is an open access article distributed under the Creative Commons Attribution License, which permits unrestricted use, distribution, and reproduction in any medium, provided the original work is properly cited.

We introduce new subclasses of close-to-convex and quasiconvex functions with respect to symmetric and conjugate points. The coefficient estimates for functions belonging to these classes are obtained.

\section{Introduction}

Let $U$ be the class of functions which are analytic and univalent in the open unit disk $E=\{z:|z|<1\}$ given by

$$
\omega(z)=\sum_{k=1}^{\infty} c_{k} z^{k}
$$

and satisfying the conditions $\omega(0)=0,|\omega(z)| \leq 1, z \in E$.

Let $S$ denote the class of functions $f$ which are analytic and univalent in $E$ of the form

$$
f(z)=z+\sum_{n=2}^{\infty} a_{n} z^{n}, \quad z \in E
$$

Let $S_{s}^{*}$ be the subclass of functions $f(z) \in S$ and satisfying the condition

$$
\operatorname{Re}\left(\frac{z f^{\prime}(z)}{f(z)-f(-z)}\right)>0, \quad z \in E
$$

These functions are called starlike with respect to symmetric points and were introduced by Sakaguchi [1].

Also, let $S_{c}^{*}$ be the subclass of functions $f(z) \in S$ and satisfying the condition

$$
\operatorname{Re}\left(\frac{z f^{\prime}(z)}{f(z)+\overline{f(\bar{z})}}\right)>0, \quad z \in E .
$$

These functions are called starlike with respect to conjugate points and were introduced by El-Ashwah and Thomas [2]. Further results on starlike functions with respect to symmetric points or conjugate points can be found in [3-5].

Then, Das and Singh [6] introduced another class $C_{s}$, namely, convex functions with respect to symmetric points and satisfying the condition

$$
\operatorname{Re}\left(\frac{\left(z f^{\prime}(z)\right)^{\prime}}{(f(z)-f(-z))^{\prime}}\right)>0, \quad z \in E .
$$

Suppose that $f$ and $g$ are two analytic functions in $E$. Then, we say that the function $g$ is subordinate to the function $f$, and we write $g(z)<f(z), z \in E$, if there exists a Schwarz function $\varpi(z)$ with $\varpi(0)=0$ and $|ळ(z)|<1$ such that $g(z)=$ $f(\omega(z)), z \in E$.

In view of subordination definition, Goel and Mehrok [7] introduced a subclass of $S_{s}^{*}$ denoted by $S_{s}^{*}(A, B)$.

Let $S_{s}^{*}(A, B)$ be the class of functions of the form (2) and satisfying the condition

$$
\frac{2 z f^{\prime}(z)}{f(z)-f(-z)}<\frac{1+A z}{1+B z}, \quad-1 \leq B<A \leq 1, \quad z \in E .
$$

Following them, many authors introduced the analogue definitions by extension as follows (see $[8,9]$ ). 
Definition 1. Let $S_{c}^{*}(A, B)$ be the subclass of $S$ consisting of functions given by (2) satisfying the condition

$$
\frac{2 z f^{\prime}(z)}{f(z)+\overline{f(\bar{z})}} \prec \frac{1+A z}{1+B z}, \quad-1 \leq B<A \leq 1, z \in E .
$$

Let $C_{s}(A, B)$ be the subclass of $S$ consisting of functions given by (2) satisfying the condition

$$
\frac{2\left(z f^{\prime}(z)\right)^{\prime}}{(f(z)-f(-z))^{\prime}} \prec \frac{1+A z}{1+B z}, \quad-1 \leq B<A \leq 1, z \in E .
$$

Let $C_{c}(A, B)$ be the subclass of $S$ consisting of functions given by (2) satisfying the condition

$$
\frac{2\left(z f^{\prime}(z)\right)^{\prime}}{\left(f(z)+\overline{f(\bar{z}))^{\prime}}\right.} \prec \frac{1+A z}{1+B z}, \quad-1 \leq B<A \leq 1, z \in E .
$$

Motivated by the pervious classes, Selvaraj and Vasanthi [10] defined the following classes of functions with respect to symmetric and conjugate points.

Definition 2. Let $M_{s}(\alpha, A, B)$ be the subclass of $S$ consisting of functions given by (2) satisfying the condition

$$
\begin{gathered}
\frac{2 z f^{\prime}(z)+2 \alpha z^{2} f^{\prime \prime}(z)}{(1-\alpha)(f(z)-f(-z))+\alpha z(f(z)-f(-z))^{\prime}} \prec \frac{1+A z}{1+B z}, \\
-1 \leq B<A \leq 1,0 \leq \alpha \leq 1, z \in E .
\end{gathered}
$$

Let $M_{c}(\alpha, A, B)$ be the subclass of $S$ consisting of functions given by (2) satisfying the condition

$$
\begin{gathered}
\frac{2 z f^{\prime}(z)+2 \alpha z^{2} f^{\prime \prime}(z)}{(1-\alpha)(f(z)+\overline{f(\bar{z})})+\alpha z(f(z)+\overline{f(\bar{z})})^{\prime}} \prec \frac{1+A z}{1+B z}, \\
-1 \leq B<A \leq 1,0 \leq \alpha \leq 1, z \in E .
\end{gathered}
$$

In this paper, we introduce the class $K_{s}^{*}(\alpha, A, B ; C, D)$ consisting of analytic functions $f$ of the form (2) and satisfying

$$
\begin{array}{r}
\frac{2 z f^{\prime}(z)+2 \alpha z^{2} f^{\prime \prime}(z)}{(1-\alpha)(g(z)-g(-z))+\alpha z(g(z)-g(-z))^{\prime}} \prec \frac{1+C z}{1+D z}, \\
-1 \leq D \leq B<A \leq C \leq 1, \\
0 \leq \alpha \leq 1, \quad z \in E,
\end{array}
$$

where $g(z)=z+\sum_{n=2}^{\infty} b_{n} z^{n} \in M_{s}(\alpha, A, B)$.
In addition, we introduce the class $K_{c}^{*}(\alpha, A, B ; C, D)$ consisting of analytic functions $f$ of the form (2) and satisfying

$$
\begin{aligned}
& \frac{2 z f^{\prime}(z)+2 \alpha z^{2} f^{\prime \prime}(z)}{(1-\alpha)(g(z)+\overline{g(\bar{z}}))+\alpha z(g(z)+\overline{g(\bar{z})})^{\prime}} \prec \frac{1+C z}{1+D z} \\
& -1 \leq D \leq B<A \leq C \leq 1, \\
& 0 \leq \alpha \leq 1, z \in E \text {, }
\end{aligned}
$$

where $g(z)=z+\sum_{n=2}^{\infty} b_{n} z^{n} \in M_{c}(\alpha, A, B)$.

We note that

(i) for $\alpha=0, K_{s}^{*}(0, A, B ; C, D)=K_{s}(A, B ; C, D)$ (see Mehrok et al. [11]) and $K_{c}^{*}(0, A, B ; C, D)=K_{c}(A$, $B ; C, D)$;

(ii) for $\alpha=0, C=1$ and $D=-1, K_{s}^{*}(0, A, B ; 1,-1)=$ $K_{s}(A, B)$ (see Janteng and Halim [12]) and $K_{c}^{*}(0, A$, $B ; 1,-1)=K_{c}(A, B)$;

(iii) for $\alpha=0, A=C=1$ and $B=D=-1, K_{s}^{*}(0,1$, $-1 ; 1,-1) \equiv K_{s}$ and $K_{c}^{*}(0,1,-1 ; 1,-1) \equiv K_{c}$;

(iv) for $\alpha=1, K_{s}^{*}(1, A, B ; C, D)=K_{s}^{*}(A, B ; C, D)$ and $K_{c}^{*}(1, A, B ; C, D)=K_{c}^{*}(A, B ; C, D) ;$

(v) for $\alpha=1, C=1$ and $D=-1, K_{s}^{*}(1, A, B ; 1,-1)=$ $K_{s}^{*}(A, B)$ (see Janteng and Halim [13]) and $K_{c}^{*}(1, A, B ; 1,-1)=K_{c}^{*}(A, B)$;

(vi) for $\alpha=1, A=C=1$ and $B=D=-1, K_{s}^{*}(1,1$, $-1 ; 1,-1) \equiv K_{s}^{*}$ and $K_{c}^{*}(1,1,-1 ; 1,-1) \equiv K_{c}^{*}$.

By the definition of subordination, it follows that $f \in$ $K_{s}^{*}(\alpha, A, B ; C, D)$ if and only if

$$
\begin{gathered}
\frac{2 z f^{\prime}(z)+2 \alpha z^{2} f^{\prime \prime}(z)}{(1-\alpha)(g(z)-g(-z))+\alpha z(g(z)-g(-z))^{\prime}} \\
=\frac{1+C \omega(z)}{1+D \omega(z)}=P(z), \quad \omega(z) \in U,
\end{gathered}
$$

and that $f \in K_{c}^{*}(\alpha, A, B ; C, D)$ if and only if

$$
\begin{gathered}
\frac{2 z f^{\prime}(z)+2 \alpha z^{2} f^{\prime \prime}(z)}{(1-\alpha)(g(z)+\overline{g(\bar{z}}))+\alpha z(g(z)+\overline{g(\bar{z})})^{\prime}} \\
=\frac{1+C \omega(z)}{1+D \omega(z)}=P(z), \quad \omega(z) \in U,
\end{gathered}
$$

where

$$
P(z)=1+\sum_{n=1}^{\infty} p_{n} z^{n}
$$

In the next section, we discuss the coefficient estimates for functions belonging to the classes $K_{s}^{*}(\alpha, A, B ; C, D)$ and $K_{c}^{*}(\alpha, A, B ; C, D)$. 


\section{Some Preliminary Lemmas}

We will require the following lemmas for proving our main results.

Lemma 3 (see [7]). If $P(z)$ is given by (14), (15) and (16), then for $-1 \leq D<C \leq 1$,

$$
\left|p_{n}\right| \leq(C-D), \quad n=1,2, \ldots
$$

Lemma 4 (see [10]). Let $g(z)=z+\sum_{n=2}^{\infty} b_{n} z^{n} \in M_{s}(\alpha, A, B)$. Then, for $n \geq 1,0 \leq \alpha \leq 1$,

$$
\begin{gathered}
\left|b_{2 n}\right| \leq \frac{(A-B)}{2^{n} \cdot n !(1+(2 n-1) \alpha)} \prod_{j=1}^{n-1}(A-B+2 j), \\
\left|b_{2 n+1}\right| \leq \frac{(A-B)}{2^{n} \cdot n !(1+2 n \alpha)} \prod_{j=1}^{n-1}(A-B+2 j) .
\end{gathered}
$$

Lemma 5 (see [10]). Let $g(z)=z+\sum_{n=2}^{\infty} b_{n} z^{n} \in M_{c}(\alpha, A, B)$ Then, for $n \geq 1,0 \leq \alpha \leq 1$,

$$
\begin{gathered}
\left|b_{2 n}\right| \leq \frac{(A-B)}{(2 n-1) !(1+(2 n-1) \alpha)} \prod_{j=1}^{2 n-2}(A-B+j), \\
\left|b_{2 n+1}\right| \leq \frac{(A-B)}{(2 n) !(1+2 n \alpha)} \prod_{j=1}^{2 n-1}(A-B+j) .
\end{gathered}
$$

\section{Main Results}

Unless otherwise mentioned, we will assume in the reminder of this paper that $-1 \leq D \leq B<A \leq C \leq 1,0 \leq \alpha \leq 1$, and $z \in E$.

Theorem 6. Let $f \in K_{s}^{*}(\alpha, A, B ; C, D)$, then for $n \geq 1$,

$$
\begin{aligned}
\left|a_{2 n}\right| \leq & \frac{(C-D)}{2^{n} \cdot n !(1+(2 n-1) \alpha)} \prod_{j=1}^{n-1}(A-B+2 j), \\
\left|a_{2 n+1}\right| \leq & \frac{1}{(2 n+1)(1+2 n \alpha)} \\
& \times\left\{\left[(C-D)+\frac{(A-B)}{2 n}\right]\right. \\
& \left.\times\left[\frac{1}{2^{n-1} \cdot(n-1) !} \prod_{j=1}^{n-1}(A-B+2 j)\right]\right\} .
\end{aligned}
$$

Proof. Since $g(z)=z+\sum_{n=2}^{\infty} b_{n} z^{n} \in M_{s}(\alpha, A, B)$, it follows that

$$
\begin{aligned}
& 2 z g^{\prime}(z)+2 \alpha z^{2} g^{\prime \prime}(z) \\
& \quad=\left[(1-\alpha)(g(z)-g(-z))+\alpha z(g(z)-g(-z))^{\prime}\right] K(z),
\end{aligned}
$$

for $z \in E$, with $\operatorname{Re}(K(z))>0$, where $K(z)=1+d_{1} z+d_{2} z^{2}+$ $d_{3} z^{3}+\cdots$.
On equating the coefficients of like powers of $z$ in (22), we get

$$
\begin{aligned}
& 2(1+\alpha) b_{2}=d_{1}, \\
& 2(1+2 \alpha) b_{3}=d_{2}, \\
& 4(1+3 \alpha) b_{4}=d_{3}+(1+2 \alpha) b_{3} d_{1}, \\
& 4(1+4 \alpha) b_{5}=d_{4}+(1+2 \alpha) b_{3} d_{2},
\end{aligned}
$$

and continuing in this way, we obtain

$$
\begin{aligned}
2 n(1+(2 n-1) \alpha) b_{2 n}= & d_{2 n-1}+(1+2 \alpha) b_{3} d_{2 n-3} \\
& +\cdots+(1+(2 n-2) \alpha) b_{2 n-1} d_{1}
\end{aligned}
$$

$$
\begin{aligned}
2 n(1+2 n \alpha) b_{2 n+1}= & d_{2 n}+(1+2 \alpha) b_{3} d_{2 n-2} \\
& +\cdots+(1+(2 n-2) \alpha) b_{2 n-1} d_{2} .
\end{aligned}
$$

From (14) and (16), we have

$$
\begin{gathered}
\left(z+2 a_{2} z^{2}+3 a_{3} z^{3}+4 a_{4} z^{4}+5 a_{5} z^{5}+\cdots+2 n a_{2 n} z^{2 n}+\cdots\right) \\
+\alpha\left(2 a_{2} z^{2}+6 a_{3} z^{3}+12 a_{4} z^{4}+20 a_{5} z^{5}+\cdots\right. \\
\left.+(2 n-1) 2 n a_{2 n} z^{2 n}+\cdots\right) \\
=\left[( 1 - \alpha ) \left(z+b_{3} z^{3}+b_{5} z^{5}+\cdots+b_{2 n-1} z^{2 n-1}\right.\right. \\
\left.+b_{2 n+1} z^{2 n+1}+\cdots\right) \\
+\alpha\left(z+3 b_{3} z^{3}+5 b_{5} z^{5}+\cdots+(2 n-1) b_{2 n-1} z^{2 n-1}\right. \\
\left.\left.+(2 n+1) b_{2 n+1} z^{2 n+1}+\cdots\right)\right] \\
\times\left(1+p_{1} z+p_{2} z^{2}+p_{3} z^{3}+p_{4} z^{4}\right. \\
\left.+p_{5} z^{5}+\cdots+p_{2 n-1} z^{2 n-1}+p_{2 n} z^{2 n}+\cdots\right) .
\end{gathered}
$$

On equating the coefficients, we obtain

$$
\begin{aligned}
& 2(1+\alpha) a_{2}=p_{1}, \\
& 3(1+2 \alpha) a_{3}=p_{2}+(1+2 \alpha) b_{3}, \\
& 4(1+3 \alpha) a_{4}=p_{3}+(1+2 \alpha) b_{3} p_{1}, \\
& 5(1+4 \alpha) a_{5}=p_{4}+(1+2 \alpha) b_{3} p_{2}+(1+4 \alpha) b_{5},
\end{aligned}
$$


and so

$$
\begin{aligned}
2 n(1 & +(2 n-1) \alpha) a_{2 n} \\
= & p_{2 n-1}+(1+2 \alpha) b_{3} p_{2 n-3} \\
& +\cdots+(1+(2 n-2) \alpha) b_{2 n-1} p_{1}, \\
& +\cdots+1)(1+2 n \alpha) a_{2 n+1} \\
= & p_{2 n}+(1+2 \alpha) b_{3} p_{2 n-2} \\
& +\cdots+(1+(2 n-2) \alpha) b_{2 n-1} p_{2}+(1+2 n \alpha) b_{2 n+1} .
\end{aligned}
$$

By using Lemma 3 and (27), we have

$$
\left|a_{2}\right| \leq \frac{(C-D)}{2 \cdot 1 \cdot(1+\alpha)}, \quad\left|a_{3}\right| \leq \frac{(A-B)+2(C-D)}{3 \cdot 2 \cdot(1+2 \alpha)} .
$$

Again, by applying Lemma 3 and using (23), we obtain from (28)

$$
\begin{aligned}
& \left|a_{4}\right| \leq \frac{(C-D)(A-B+2)}{4 \cdot 2 \cdot(1+3 \alpha)} \\
& \left|a_{5}\right| \leq \frac{(A-B+2)[(A-B)+4(C-D)]}{5 \cdot 8 \cdot(1+4 \alpha)} .
\end{aligned}
$$

It follows that (20) and (21) hold for $n=1,2$. We now prove (20) and (21) by induction.

Equation (29) together with Lemma 3 yield

$$
\begin{aligned}
\left|a_{2 n}\right| \leq & \frac{(C-D)}{2 n(1+(2 n-1) \alpha)}\left[1+\sum_{k=1}^{n-1}(1+2 k \alpha)\left|b_{2 k+1}\right|\right] \\
\left|a_{2 n+1}\right| \leq & \frac{1}{(2 n+1)(1+2 n \alpha)} \\
& \times\left\{(C-D)\left[1+\sum_{k=1}^{n-1}(1+2 k \alpha)\left|b_{2 k+1}\right|\right]\right. \\
& \left.+(1+2 n \alpha)\left|b_{2 n+1}\right|\right\} .
\end{aligned}
$$

Again, using Lemma 3 in (25), we have

$$
\left|b_{2 n+1}\right| \leq \frac{(A-B)}{2 n(1+2 n \alpha)}\left[1+\sum_{k=1}^{n-1}(1+2 k \alpha)\left|b_{2 k+1}\right|\right] .
$$

Using (34) in (33), we obtain

$\left|a_{2 n+1}\right|$

$$
\begin{aligned}
\leq & \frac{1}{(2 n+1)(1+2 n \alpha)} \\
& \times\left\{\left[(C-D)+\frac{(A-B)}{2 n}\right]\left[1+\sum_{k=1}^{n-1}(1+2 k \alpha)\left|b_{2 k+1}\right|\right]\right\} .
\end{aligned}
$$

We suppose that (20) and (21) hold for $k=3,4, \ldots,(n-1)$.
Using Lemma 4 in (32) and (35), we get

$$
\begin{aligned}
\left|a_{2 n}\right| \leq & \frac{(C-D)}{2 n(1+(2 n-1) \alpha)} \\
& \times\left[1+\sum_{k=1}^{n-1} \frac{(A-B)}{2^{k} \cdot k !} \prod_{j=1}^{k-1}(A-B+2 j)\right],
\end{aligned}
$$

$$
\begin{aligned}
\left|a_{2 n+1}\right| \leq & \frac{1}{(2 n+1)(1+2 n \alpha)} \\
& \times\left\{\left[(C-D)+\frac{(A-B)}{2 n}\right]\right. \\
& \left.\times\left[1+\sum_{k=1}^{n-1} \frac{(A-B)}{2^{k} \cdot k !} \prod_{j=1}^{k-1}(A-B+2 j)\right]\right\} .
\end{aligned}
$$

In order to prove (20), it is sufficient to show that

$$
\begin{gathered}
\frac{(C-D)}{2 m(1+(2 m-1) \alpha)}\left[1+\sum_{k=1}^{m-1} \frac{(A-B)}{2^{k} \cdot k !} \prod_{j=1}^{k-1}(A-B+2 j)\right] \\
=\frac{(C-D)}{2^{m} \cdot m !(1+(2 m-1) \alpha)} \prod_{j=1}^{m-1}(A-B+2 j) \\
(m=3,4, \ldots, n) .
\end{gathered}
$$

Thus, (38) is valid for $m=3$.

Let us assume that (38) is true for all $m, 3<m \leq(n-1)$. Then, from (36), we have

$$
\begin{aligned}
& \frac{(C-D)}{2 n(1+(2 n-1) \alpha)}\left[1+\sum_{k=1}^{n-1} \frac{(A-B)}{2^{k} \cdot k !} \prod_{j=1}^{k-1}(A-B+2 j)\right] \\
& =\frac{(n-1)}{n}\left\{\frac{(C-D)}{2(n-1)(1+(2 n-1) \alpha)}\right. \\
& \left.\quad \times\left[1+\sum_{k=1}^{n-2} \frac{(A-B)}{2^{k} \cdot k !} \prod_{j=1}^{k-1}(A-B+2 j)\right]\right\} \\
& +\frac{(C-D)}{2 n(1+(2 n-1) \alpha)} \cdot \frac{(A-B)}{2^{n-1} \cdot(n-1) !} \\
& \quad \times \prod_{j=1}^{n-2}(A-B+2 j)
\end{aligned}
$$




$$
\begin{aligned}
= & \frac{(n-1)}{n} \cdot \frac{(C-D)}{2^{n-1} \cdot(n-1) !(1+(2 n-1) \alpha)} \\
& \times \prod_{j=1}^{n-2}(A-B+2 j) \\
& +\frac{(C-D)}{2 n(1+(2 n-1) \alpha)} \cdot \frac{(A-B)}{2^{n-1} \cdot(n-1) !} \\
& \times \prod_{j=1}^{n-2}(A-B+2 j) \\
= & \frac{(C-D)}{2^{n} \cdot n !(1+(2 n-1) \alpha)} \prod_{j=1}^{n-2}(A-B+2 j) \\
& \times(A-B+2(n-1)) \\
= & \frac{(C-D)}{2^{n} \cdot n !(1+(2 n-1) \alpha)} \prod_{j=1}^{n-1}(A-B+2 j) .
\end{aligned}
$$

Thus, (38) holds for $m=n$, and, hence, (20) follows. Next, we prove (21).

From (38), we have

$$
\begin{aligned}
1+\sum_{k=1}^{n-1} \frac{(A-B)}{2^{k} \cdot k !} \prod_{j=1}^{k-1}(A-B+2 j) \\
\quad=\frac{1}{2^{n-1} \cdot(n-1) !} \prod_{j=1}^{n-1}(A-B+2 j) .
\end{aligned}
$$

By using (40) in (37), we obtain

$$
\begin{aligned}
\left|a_{2 n+1}\right| \leq & \frac{1}{(2 n+1)(1+2 n \alpha)} \\
& \times\left\{\left[(C-D)+\frac{(A-B)}{2 n}\right]\right. \\
& \left.\times\left[\frac{1}{2^{n-1} \cdot(n-1) !} \prod_{j=1}^{n-1}(A-B+2 j)\right]\right\}
\end{aligned}
$$

which proves (21)

By taking $\alpha=0$ and $\alpha=1$ in Theorem 6, respectively, we can readily deduce the following corollaries.
Corollary 7 (see [11]). Let $f \in K_{s}(A, B ; C, D)$, then, for $n \geq 1$,

$$
\begin{aligned}
\left|a_{2 n}\right| \leq \frac{(C-D)}{2^{n} \cdot n !} \prod_{j=1}^{n-1}(A-B+2 j) & \\
\left|a_{2 n+1}\right| \leq \frac{1}{2 n+1}\{ & {\left[(C-D)+\frac{(A-B)}{2 n}\right] } \\
& \left.\times\left[\frac{1}{2^{n-1} \cdot(n-1) !} \prod_{j=1}^{n-1}(A-B+2 j)\right]\right\} .
\end{aligned}
$$

Corollary 8. Let $f \in K_{s}^{*}(A, B ; C, D)$, then, for $n \geq 1$,

$$
\left|a_{2 n}\right| \leq \frac{(C-D)}{2^{n+1} \cdot n \cdot n !} \prod_{j=1}^{n-1}(A-B+2 j)
$$

$\left|a_{2 n+1}\right| \leq \frac{1}{(2 n+1)^{2}}\left\{\left[(C-D)+\frac{(A-B)}{2 n}\right]\right.$

$$
\left.\times\left[\frac{1}{2^{n-1} \cdot(n-1) !} \prod_{j=1}^{n-1}(A-B+2 j)\right]\right\} .
$$

Further, putting $C=1$ and $D=-1$ in Corollary 8,one obtains the following.

Corollary 9. Let $f \in K_{s}^{*}(A, B)$, then, for $n \geq 1$,

$$
\begin{aligned}
\left|a_{2 n}\right| \leq & \frac{1}{2^{n} \cdot n \cdot n !} \prod_{j=1}^{n-1}(A-B+2 j) \\
\left|a_{2 n+1}\right| \leq & \frac{1}{(2 n+1)^{2}} \\
& \times\left\{\left[2+\frac{(A-B)}{2 n}\right]\right. \\
& \left.\times\left[\frac{1}{2^{n-1} \cdot(n-1) !} \prod_{j=1}^{n-1}(A-B+2 j)\right]\right\}
\end{aligned}
$$

Remark 10. Corollary 9 improves the result obtained by Janteng and Halim [13, Theorem 3.1]. 
Theorem 11. Let $f \in K_{c}^{*}(\alpha, A, B ; C, D)$, then, for $n \geq 1$,

$$
\begin{aligned}
\left|a_{2 n}\right| \leq & \frac{1}{2 n(1+(2 n-1) \alpha)} \\
\times & \left\{\left[(C-D)+\frac{(A-B)}{2 n-1}\right]\left[\frac{1}{(2 n-2) !} \prod_{j=1}^{2 n-2}(A-B+j)\right]\right\}, \\
\left|a_{2 n+1}\right| \leq & \frac{1}{(2 n+1)(1+2 n \alpha)} \\
& \times\left\{\left[(C-D)+\frac{(A-B)}{2 n}\right]\left[\frac{1}{(2 n-1) !} \prod_{j=1}^{2 n-1}(A-B+j)\right]\right\} .
\end{aligned}
$$

Proof. Since $g(z)=z+\sum_{n=2}^{\infty} b_{n} z^{n} \in M_{c}(\alpha, A, B)$, it follows that

$$
\begin{aligned}
& 2 z g^{\prime}(z)+2 \alpha z^{2} g^{\prime \prime}(z) \\
& \quad=\left[(1-\alpha)(g(z)+\overline{g(\bar{z}})+\alpha z(g(z)+\overline{g(\bar{z})})^{\prime}\right] K(z),
\end{aligned}
$$

where $K(z)=1+d_{1} z+d_{2} z^{2}+d_{3} z^{3}+\cdots$.

On equating the coefficients of like powers of $z$ in (47), we get

$$
\begin{gathered}
(1+\alpha) b_{2}=d_{1}, \\
2(1+2 \alpha) b_{3}=d_{2}+(1+\alpha) b_{2} d_{1}, \\
3(1+3 \alpha) b_{4}=d_{3}+(1+\alpha) b_{2} d_{2}+(1+2 \alpha) b_{3} d_{1} \\
4(1+4 \alpha) b_{5}=d_{4}+(1+\alpha) b_{2} d_{3}+(1+2 \alpha) b_{3} d_{2} \\
+(1+3 \alpha) b_{4} d_{1}
\end{gathered}
$$

and continuing in this way, we obtain

$$
\begin{aligned}
& (2 n-1)(1+(2 n-1) \alpha) b_{2 n} \\
& \quad=d_{2 n-1}+(1+\alpha) b_{2} d_{2 n-2}+\cdots+(1+(2 n-2) \alpha) b_{2 n-1} d_{1}
\end{aligned}
$$

$$
\begin{aligned}
& 2 n(1+2 n \alpha) b_{2 n+1} \\
& \quad=d_{2 n}+(1+\alpha) b_{2} d_{2 n-1}+\cdots+(1+(2 n-1) \alpha) b_{2 n} d_{1} .
\end{aligned}
$$

From (15) and (16), we have

$$
\begin{aligned}
& \left(z+2 a_{2} z^{2}+3 a_{3} z^{3}+4 a_{4} z^{4}+5 a_{5} z^{5}+\cdots+2 n a_{2 n} z^{2 n}+\cdots\right) \\
& +\alpha\left(2 a_{2} z^{2}+6 a_{3} z^{3}+12 a_{4} z^{4}+20 a_{5} z^{5}\right. \\
& \left.+\cdots+(2 n-1) 2 n a_{2 n} z^{2 n}+\cdots\right) \\
& =\left[( 1 - \alpha ) \left(z+b_{2} z^{2}+b_{3} z^{3}\right.\right. \\
& \left.+b_{4} z^{4}+b_{5} z^{5}+\cdots+b_{2 n} z^{2 n}+\cdots\right) \\
& +\alpha\left(z+2 b_{2} z^{2}+3 b_{3} z^{3}\right. \\
& \left.\left.+4 b_{4} z^{4}+5 b_{5} z^{5}+\cdots+2 n b_{2 n} z^{2 n}+\cdots\right)\right] \\
& \times\left(1+p_{1} z+p_{2} z^{2}+p_{3} z^{3}\right. \\
& \left.+p_{4} z^{4}+p_{5} z^{5}+\cdots+p_{2 n} z^{2 n}+\cdots\right) .
\end{aligned}
$$

On equating the coefficients, we obtain

$$
\begin{aligned}
2(1+\alpha) a_{2}= & p_{1}+(1+\alpha) b_{2}, \\
3(1+2 \alpha) a_{3}= & p_{2}+(1+\alpha) b_{2} p_{1} \\
& +(1+2 \alpha) b_{3}, \\
4(1+3 \alpha) a_{4}= & p_{3}+(1+\alpha) b_{2} p_{2}+(1+2 \alpha) b_{3} p_{1} \\
& +(1+3 \alpha) b_{4}, \\
5(1+4 \alpha) a_{5}= & p_{4}+(1+\alpha) b_{2} p_{3}+(1+2 \alpha) b_{3} p_{2} \\
& +(1+3 \alpha) b_{4} p_{1}+(1+4 \alpha) b_{5},
\end{aligned}
$$

and so

$$
\begin{aligned}
2 n(1+(2 n-1) \alpha) a_{2 n}= & p_{2 n-1}+(1+\alpha) b_{2} p_{2 n-2} \\
& +(1+2 \alpha) b_{3} p_{2 n-3}+\cdots \\
& +(1+(2 n-2) \alpha) b_{2 n-1} p_{1} \\
& +(1+(2 n-1) \alpha) b_{2 n} \\
(2 n+1)(1+2 n \alpha) a_{2 n+1}= & p_{2 n}+(1+\alpha) b_{2} p_{2 n-1} \\
& +(1+2 \alpha) b_{3} p_{2 n-2}+\cdots \\
& +(1+(2 n-1) \alpha) b_{2 n} p_{1} \\
& +(1+2 n \alpha) b_{2 n+1} .
\end{aligned}
$$

By using Lemma 3, (48), and (54), we have

$$
\begin{aligned}
& \left|a_{2}\right| \leq \frac{(C-D)+(A-B)}{2 \cdot 1 \cdot(1+\alpha)}, \\
& \left|a_{3}\right| \leq \frac{(A-B+1)[(A-B)+2(C-D)]}{3 \cdot 2 \cdot(1+2 \alpha)} .
\end{aligned}
$$


Again, by applying Lemma 3 and using (48)-(50), we obtain from (55) and (56)

$$
\begin{aligned}
\left|a_{4}\right| \leq & \frac{(A-B+1)(A-B+2)[(A-B)+3(C-D)]}{4 \cdot 6 \cdot(1+3 \alpha)} \\
\left|a_{5}\right| \leq & (A-B+1)(A-B+2)(A-B+3) \\
& \times[(A-B)+4(C-D)](5 \cdot 24 \cdot(1+4 \alpha))^{-1} .
\end{aligned}
$$

It follows that (45) and (46) hold for $n=1,2$. We now prove (45) by induction.

Equation (57) together with Lemma 3 yield

$$
\begin{aligned}
\left|a_{2 n}\right| \leq & \frac{1}{2 n(1+(2 n-1) \alpha)} \\
& \times\left\{( C - D ) \left[1+\sum_{k=1}^{n-1}(1+(2 k-1) \alpha)\left|b_{2 k}\right|\right.\right. \\
& \left.+\sum_{k=1}^{n-1}(1+2 k \alpha)\left|b_{2 k+1}\right|\right] \\
& \left.+(1+(2 n-1) \alpha)\left|b_{2 n}\right|\right\} .
\end{aligned}
$$

Again, by using Lemma 3 in (51), we have

$$
\begin{aligned}
\left|b_{2 n}\right| \leq & \frac{(A-B)}{(2 n-1)(1+(2 n-1) \alpha)} \\
\times & {\left[1+\sum_{k=1}^{n-1}(1+(2 k-1) \alpha)\left|b_{2 k}\right|\right.} \\
& \left.+\sum_{k=1}^{n-1}(1+2 k \alpha)\left|b_{2 k+1}\right|\right]
\end{aligned}
$$

Using (62) in (61), we obtain

$$
\begin{aligned}
\left|a_{2 n}\right| \leq & \frac{1}{2 n(1+(2 n-1) \alpha)} \\
& \times\left\{\left[(C-D)+\frac{(A-B)}{2 n-1}\right]\right. \\
& \times\left[1+\sum_{k=1}^{n-1}(1+(2 k-1) \alpha)\left|b_{2 k}\right|\right. \\
& \left.\left.+\sum_{k=1}^{n-1}(1+2 k \alpha)\left|b_{2 k+1}\right|\right]\right\} .
\end{aligned}
$$

We suppose that (45) holds for $k=3,4, \ldots,(n-1)$.
Using Lemma 5 in (63), we get

$$
\begin{aligned}
&\left|a_{2 n}\right| \leq \frac{1}{2 n(1+(2 n-1) \alpha)} \\
& \times\left\{\left[(C-D)+\frac{(A-B)}{2 n-1}\right]\right. \\
& \times\left[1+\sum_{k=1}^{n-1} \frac{(A-B)}{(2 k-1) !} \prod_{j=1}^{2 k-2}(A-B+j)\right. \\
&\left.\left.\quad+\sum_{k=1}^{n-1} \frac{(A-B)}{(2 k) !} \prod_{j=1}^{2 k-1}(A-B+j)\right]\right\} .
\end{aligned}
$$

In order to prove (45), it is sufficient to show that

$$
\begin{gathered}
\frac{1}{2 m(1+(2 m-1) \alpha)} \\
\times\left\{\left[(C-D)+\frac{(A-B)}{2 m-1}\right]\right. \\
\times\left[1+\sum_{k=1}^{m-1} \frac{(A-B)}{(2 k-1) !} \prod_{j=1}^{2 k-2}(A-B+j)\right. \\
=\frac{\left.\left(+\sum_{k=1}^{m-1} \frac{(A-B)}{(2 k) !} \prod_{j=1}^{2 k-1}(A-B+j)\right]\right\}}{2 m(1+(2 m-1) \alpha)} \\
\times\left\{\left[(C-D)+\frac{(A-B)}{2 m-1}\right]\right. \\
\left.\times\left[\frac{1}{(2 m-2) !} \prod_{j=1}^{2 m-2}(A-B+j)\right]\right\}
\end{gathered}
$$

Thus, (65) is valid for $m=3$.

Let us assume that (65) is true for all $m, 3<m \leq(n-1)$. Then, from (64), we have

$$
\begin{aligned}
& \frac{1}{2 n(1+(2 n-1) \alpha)} \\
& \times\left\{\sum_{k=1}^{n-1}\left[(C-D)+\frac{(A-B)}{2 n-1}\right]\right. \\
& \times\left[1+\sum_{k=1}^{n-1} \frac{(A-B)}{(2 k-1) !} \prod_{j=1}^{2 k-2}(A-B+j)\right. \\
& \left.\left.+\sum_{k=1}^{n-1} \frac{(A-B)}{(2 k) !} \prod_{j=1}^{2 k-1}(A-B+j)\right]\right\}
\end{aligned}
$$




$$
\begin{aligned}
& =\left(\frac{n-1}{n}\right)\left\{\sum_{k=1}^{n-2} \frac{1}{2(n-1)(1+(2 n-1) \alpha)}\right. \\
& \times\left[(C-D)+\frac{(A-B)}{2 n-1}\right] \\
& \times\left[1+\sum_{k=1}^{n-2} \frac{(A-B)}{(2 k-1) !} \prod_{j=1}^{2 k-2}(A-B+j)\right. \\
& \left.\left.+\sum_{k=1}^{n-2} \frac{(A-B)}{(2 k) !} \prod_{j=1}^{2 k-1}(A-B+j)\right]\right\} \\
& +\frac{1}{2 n(1+(2 n-1) \alpha)}\left[(C-D)+\frac{(A-B)}{2 n-1}\right] \\
& \times\left[\frac{(A-B)}{(2(n-1)-1) !} \prod_{j=1}^{2 n-4}(A-B+j)\right. \\
& \left.+\frac{(A-B)}{(2(n-1)) !} \prod_{j=1}^{2 n-3}(A-B+j)\right] \\
& =\left(\frac{n-1}{n}\right) \cdot \frac{1}{2(n-1)(1+(2 n-1) \alpha)} \\
& \times\left[(C-D)+\frac{(A-B)}{2 n-1}\right] \\
& \times\left[\frac{1}{(2(n-1)-2) !} \prod_{j=1}^{2 n-4}(A-B+j)\right] \\
& +\frac{1}{2 n(1+(2 n-1) \alpha)}\left[(C-D)+\frac{(A-B)}{2 n-1}\right] \\
& \times\left[\frac{(A-B)}{(2(n-1)-1) !} \prod_{j=1}^{2 n-4}(A-B+j)\right. \\
& \left.+\frac{(A-B)}{(2(n-1)) !} \prod_{j=1}^{2 n-3}(A-B+j)\right] \\
& =\frac{1}{2 n(1+(2 n-1) \alpha)}\left[(C-D)+\frac{(A-B)}{2 n-1}\right] \\
& \times\left[\frac{1}{(2(n-1)-1) !} \prod_{j=1}^{2 n-4}(A-B+j)\right. \\
& \times(A-B+(2 n-3)) \\
& \left.+\frac{(A-B)}{(2(n-1)) !} \prod_{j=1}^{2 n-3}(A-B+j)\right]
\end{aligned}
$$

$$
\begin{aligned}
= & \frac{1}{2 n(1+(2 n-1) \alpha)}\left[(C-D)+\frac{(A-B)}{2 n-1}\right] \\
& \times\left[\frac{1}{(2(n-1)-1) !} \prod_{j=1}^{2 n-3}(A-B+j)\right. \\
& \left.+\frac{(A-B)}{(2(n-1)) !} \prod_{j=1}^{2 n-3}(A-B+j)\right] \\
= & \frac{1}{2 n(1+(2 n-1) \alpha)}\left[(C-D)+\frac{(A-B)}{2 n-1}\right] \\
& \times\left[\frac{1}{(2(n-1)) !} \prod_{j=1}^{2 n-3}(A-B+j)\right. \\
= & \times\left[\frac{1}{2 n(1+(2 n-1) \alpha)}\left[(C-D)+\frac{(A-B)}{2 n-1}\right]\right. \\
& \times(A-B+(2 n-2))] \\
& {\left[\begin{array}{l}
2 n-2 \\
(2 n-1
\end{array}\right] }
\end{aligned}
$$

Thus, (65) holds for $m=n$, and, hence, (45) follows. Similarly, we can prove (46).

By taking $\alpha=0$ and $\alpha=1$ in Theorem 11 , respectively, we can easily derive the following corollaries.

Corollary 12. Let $f \in K_{c}(A, B ; C, D)$, then, for $n \geq 1$,

$$
\begin{aligned}
&\left|a_{2 n}\right| \leq \frac{1}{2 n}\{[\left.(C-D)+\frac{(A-B)}{2 n-1}\right] \\
& \times {\left.\left[\frac{1}{(2 n-2) !} \prod_{j=1}^{2 n-2}(A-B+j)\right]\right\}, } \\
&\left|a_{2 n+1}\right| \leq \frac{1}{2 n+1}\left\{\left[(C-D)+\frac{(A-B)}{2 n}\right]\right. \\
&\left.\times\left[\frac{1}{(2 n-1) !} \prod_{j=1}^{2 n-1}(A-B+j)\right]\right\} .
\end{aligned}
$$


Corollary 13. Let $f \in K_{c}^{*}(A, B ; C, D)$, then, for $n \geq 1$,

$$
\begin{aligned}
& \left|a_{2 n}\right| \leq \frac{1}{(2 n)^{2}}\left\{\left[(C-D)+\frac{(A-B)}{2 n-1}\right]\right. \\
& \left.\times\left[\frac{1}{(2 n-2) !} \prod_{j=1}^{2 n-2}(A-B+j)\right]\right\}, \\
& \left|a_{2 n+1}\right| \leq \frac{1}{(2 n+1)^{2}}\left\{\left[(C-D)+\frac{(A-B)}{2 n}\right]\right. \\
& \left.\times\left[\frac{1}{(2 n-1) !} \prod_{j=1}^{2 n-1}(A-B+j)\right]\right\}
\end{aligned}
$$

\section{Acknowledgments}

The present investigation was partly supported by the Natural Science Foundation of China under Grant no. 11271045, the Higher School Doctoral Foundation of China under Grant no. 20100003110004, and the Natural Science Foundation of Inner Mongolia of China under Grant no. 2010MS0117. The authors would like to thank Professor Jacek Dziok for his valuable suggestions and the referees for their careful reading and helpful comments to improve the paper.

\section{References}

[1] K. Sakaguchi, “On certain univalent mappings," Journal of the Mathematical Society of Japan, vol. 11, pp. 72-75, 1959.

[2] R. M. El-Ashwah and D. K. Thomas, "Some subclasses of closeto-convex functions," Journal of Ramanujan Mathematical Society, vol. 2, pp. 86-100, 1987.

[3] J. Sokol, "Some remarks on the class of functions starlike with respect to symmetric points," Folia Scientiarum Universitatis Technicae Resoviensis, vol. 73, pp. 79-91, 1990.

[4] J. Sokol, "Function starlike with respect to conjugate points," Folia Scientiarum Universitatis Technicae Resoviensis, vol. 85, pp. 53-65, 1991.

[5] J. Sokol, A. Szpila, and M. Szpila, "On some subclass of starlike functions with respect to symmetric points," Folia Scientiarum Universitatis Technicae Resoviensis, vol. 85, pp. 65-73, 1991.

[6] R. N. Das and P. Singh, "On subclasses of schlicht mapping," Indian Journal of Pure and Applied Mathematics, vol. 8, pp. 864-872, 1977.

[7] R. M. Goel and B. C. Mehrok, "A subclass of starlike functions with respect to symmetric points," Tamkang Journal of Mathematics, vol. 13, no. 1, pp. 11-24, 1982.

[8] S. A. F. M. Dahhar and A. Janteng, "A subclass of starlike functions with respect to conjugate points," International Mathematical Forum, vol. 28, no. 4, pp. 1373-1377, 2009.

[9] A. Janteng and S. A. Halim, "A subclass of convex functions with respect to symmetric points," in Proceedings of The 16th National Symposium on Science Mathematical, 2008.

[10] C. Selvaraj and N. Vasanthi, "Subclasses of analytic functionswith respect to symmetric and conjugate points," Tamkang Journal of Mathematics, vol. 42, no. 1, pp. 87-94, 2011.
[11] B. S. Mehrok, G. Singh, and D. Gupta, "Coefficient estimate for a subclass of close-to-convex functions with respect to symmetric points," Tamkang Journal of Mathematics, vol. 42, no. 2, pp. 217-222, 2011.

[12] A. Janteng and S. A. Halim, "Coefficient estimate for a subclass of close-to-convex functions with respect to symmetric points," International Journal of Mathematical Analysis, vol. 3, no. 5-8, pp. 309-313, 2009.

[13] A. Janteng and S. Abdul Halim, "A subclass of quasi-convex functions with respect to symmetric points," Applied Mathematical Sciences, vol. 3, no. 12, pp. 551-556, 2009. 


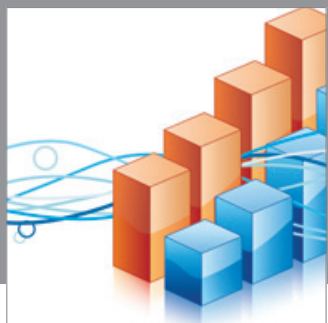

Advances in

Operations Research

mansans

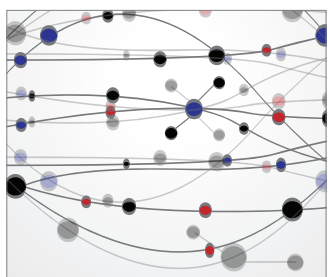

The Scientific World Journal
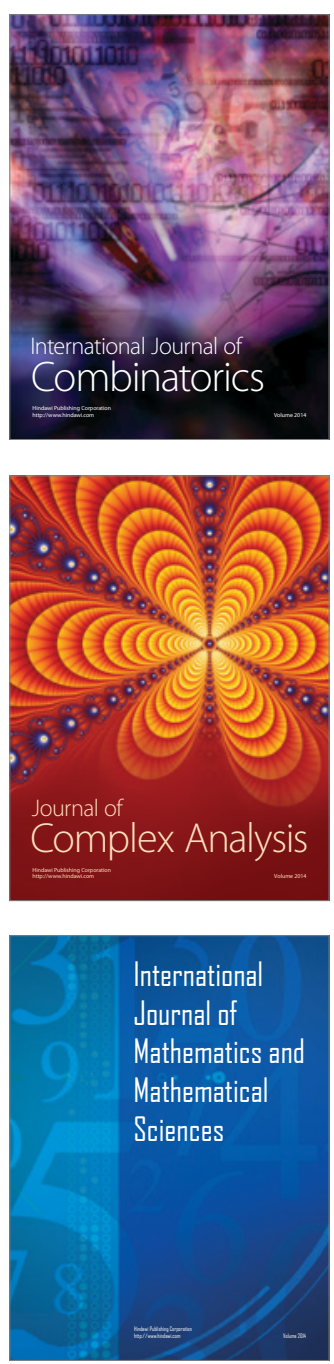
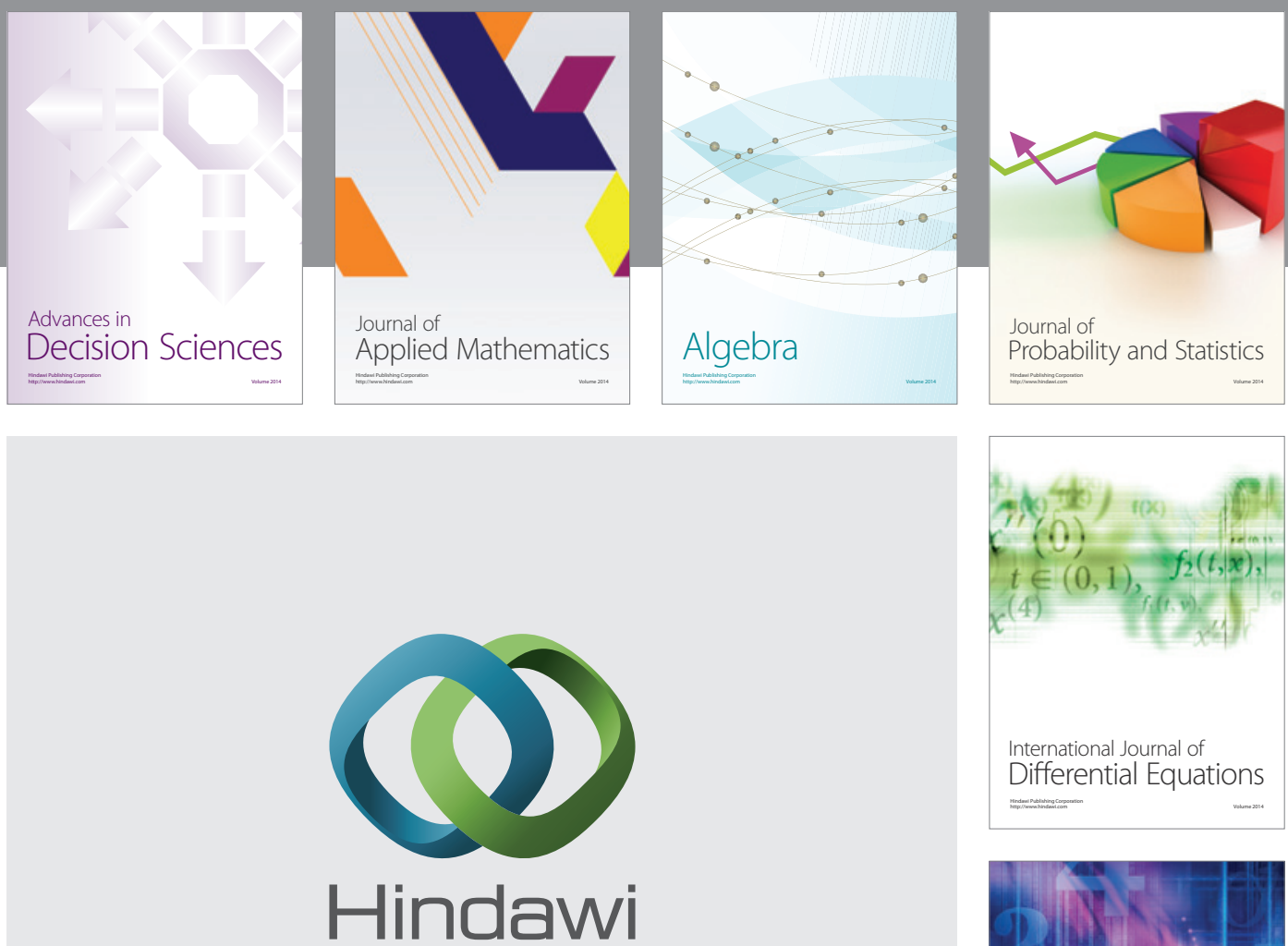

Submit your manuscripts at http://www.hindawi.com
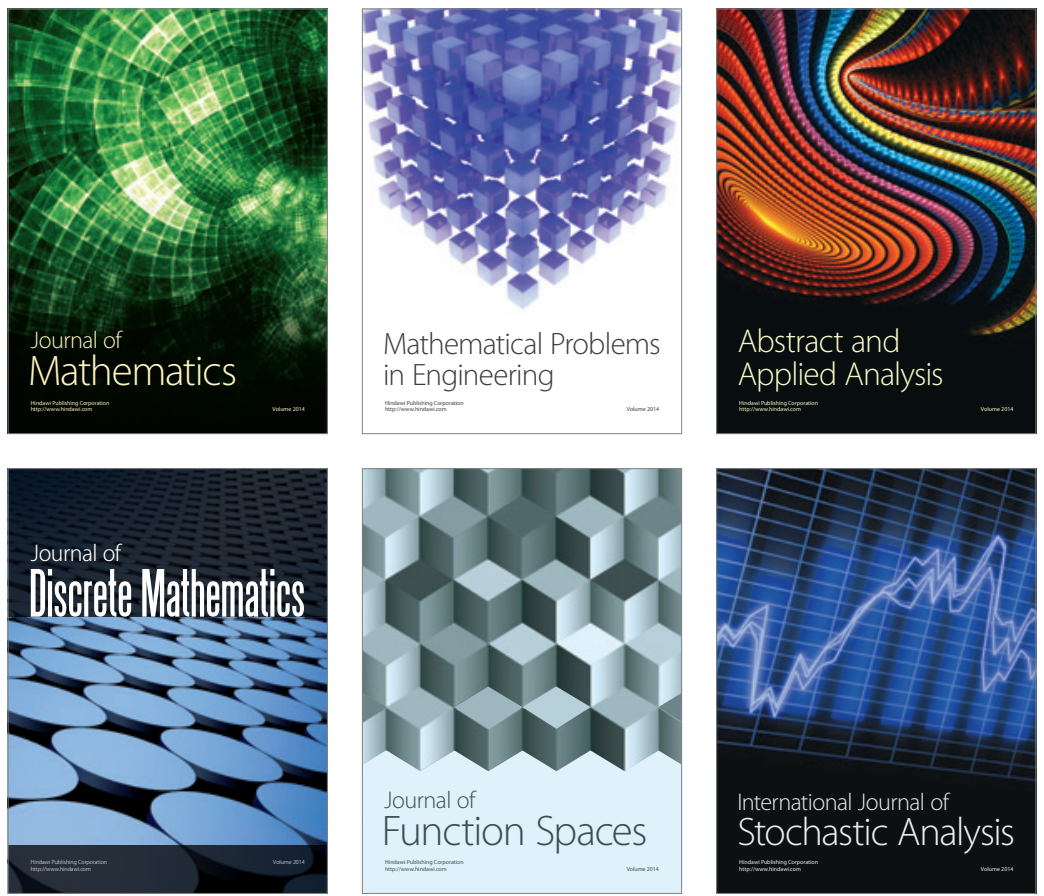

Journal of

Function Spaces

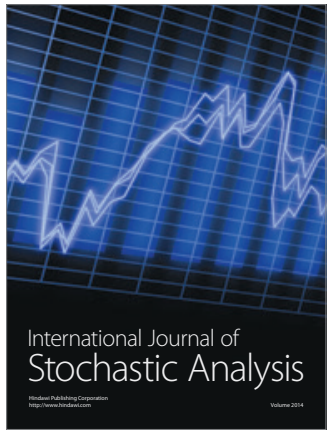

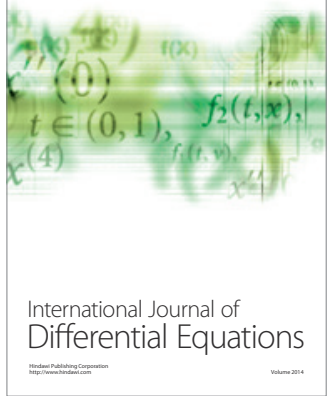
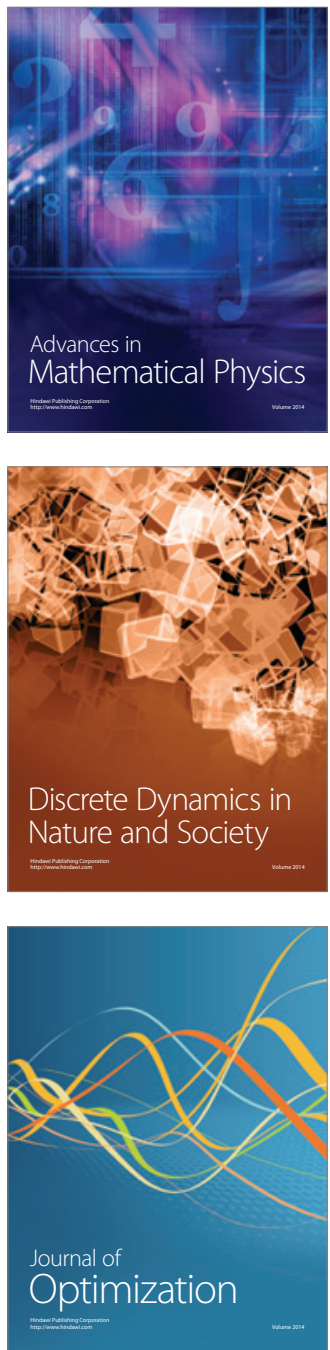